PROCEEDINGS OF THE

AMERICAN MATHEMATICAL SOCIETY

Volume 126, Number 11, November 1998, Pages 3243-3252

S 0002-9939(98)04370-6

\title{
UNBOUNDED DOMAINS OF NORMALITY
}

\author{
J. M. ANDERSON AND A. HINKKANEN
}

(Communicated by Albert Baernstein II)

\begin{abstract}
Let $f$ be a transcendental entire function of order less than $1 / 2$. We introduce the method of "self-sustaining spread" to study the components of the set of normality of such a function. We give a new proof of the fact that any preperiodic or periodic component of the set of normality of $f$ is bounded. We obtain the same conclusion for a wandering domain if the growth rate of $f$ is never too small.
\end{abstract}

\section{INTRODUCTION}

Let $f$ be a transcendental entire function. The set of normality (or Fatou set) $N(f)$ of $f$ consists, by definition, of all $z$ in the complex plane $\mathbb{C}$ that have a neighborhood $U$ such that the family $\left\{f^{n} \mid U: n \geq 1\right\}$ of the restrictions of the iterates of $f$ to $U$ is a normal family. Here $f^{1}=f$, and $f^{n}=f \circ f^{n-1}$ for $n \geq 2$. The Julia set $J(f)$ of $f$ is defined by $J(f)=\mathbb{C} \backslash N(f)$. Clearly $N(f)$ is open, while by the results of Fatou and of Julia, $J(f)$ is a nonempty perfect set which coincides with $\mathbb{C}$, or is nowhere dense in $\mathbb{C}$. For the basic results in the iteration theory of rational and entire functions based on these concepts we refer to the original works of Fatou [8, 9, 10] and of Julia [11] and to the book of Beardon [6].

Let $D$ be a component of $N(f)$. Then $f^{n}(D) \subset D_{n}$ where $D_{n}$ is a component of $N(f)$. If all the $D_{n}$ 's are disjoint, then $D$ is called a wandering domain. If there is a smallest positive integer $p$ such that $D_{p}=D$, then $D$ is periodic of period $p$. In particular, if $p=1$, then $D$ is called invariant. Otherwise, $D$ is called preperiodic, and then $D_{n}=D_{n+p}$ for some $n, p \geq 1$, while $D_{p} \neq D$ for all $p \geq 1$.

Baker [3] raised the question of whether every component of $N(f)$ must be bounded if $f$ is of sufficiently small growth. The appropriate growth condition would appear to be of order 1/2, minimal type at most. Baker [3, p. 489] noted that for any sufficiently large positive $a$, the function $f(z)=z^{-1 / 2} \sin \sqrt{z}+z+a$ is of order $1 / 2$, mean type, and has an unbounded component $D$ of $N(f)$ containing, in fact, a segment $\left[x_{0}, \infty\right)$ of the positive real axis, such that $f^{n}(z) \rightarrow \infty$ as $n \rightarrow \infty$, locally uniformly in $D$. In the positive direction, Baker proved the following result [3, Theorem 1, p. 484, Lemma 7, p. 490].

Received by the editors July 8, 1996 and, in revised form, March 10, 1997.

1991 Mathematics Subject Classification. Primary 30D05, 58F23.

This research was completed while the first author was visiting the University of Illinois at Urbana-Champaign. He wishes to thank the Department of Mathematics for its kind hospitality.

The research of the second author was partially supported by the Alfred P. Sloan Foundation, by the U.S. National Science Foundation grant DMS 94-00999, and by the U.S. National Security Agency grant MDA904-95-H-1014.

(C)1998 American Mathematical Society 
Theorem A. Let $f$ be a transcendental entire function of growth not exceeding order $1 / 2$, minimal type. Let $D$ be a component of $N(f)$ such that either

(i) all limit functions of convergent subsequences of iterates of $f$ are finite in $D$; or

(ii) there is a component $U$ of $N(f)$ and an integer $m \geq 0$ such that $f^{m}(D) \subset U$ and $f(U) \subset U$, and furthermore $f^{n}(z) \rightarrow \infty$ as $n \rightarrow \infty$, locally uniformly for $z \in U$.

Then $D$ is bounded.

More precisely, note first that if the component $D$ of $N(f)$ is unbounded and if $f$ is as in Theorem A, then by Theorem B below, the minimum modulus function of $f$ is unbounded so that $f(D)$, and hence, by induction, $f^{n}(D)$ for each $n \geq 1$, is unbounded. So, if $D$ is not a wandering domain, we may assume that $D$ is periodic, that is, $f^{p}(D) \subset D$ for some $p \geq 1$. Now if all limit functions of convergent subsequences of iterates of $f$ are finite in $D$, then the conclusion of Theorem A follows from [3, Lemma 7, p. 490]. Otherwise, if $D$ is not a wandering domain, so that we may assume that $f^{p}(D) \subset D$, we note that $f^{n}(z) \rightarrow \infty$ in $D$ (otherwise all limit functions are finite by the classification of periodic domains). When $D$ is invariant, the conclusion of Theorem A follows from [3, Theorem 1, p. 484]. Concerning the case $p \geq 2$, we obtain a new proof of the following result.

Theorem 1. Let $f$ be a transcendental entire function of order less than $1 / 2$. Then every preperiodic or periodic component of the set of normality $N(f)$ of $f$ is bounded.

When $p=1$ and $f^{n} \rightarrow \infty$ in $D$, Baker's proof of Theorem A (ii) is based on the fact that $D$ contains a path $\gamma$ going to infinity such that $|f(z)|=O\left(|z|^{q}\right)$ for some $q>0$ as $z \rightarrow \infty$ along $\gamma$. This contradicts Theorem B below. When $p \geq 2$, the same argument shows that $\left|f^{p}(z)\right|=O\left(|z|^{q}\right)$ on $\gamma$. Since, in general, $f^{p}$ is of order $>1 / 2$, no immediate contradiction is obtained, so that a more detailed analysis is required.

Theorem 1 has been proved by Stallard [12, Theorem 3A, p. 49], in her Ph.D. thesis, but her proof, which relies on a theorem of Baker [2, Lemma 1, p. 148] (whose proof, in turn, uses the proof of [1, Satz 2, p. 129]), remains unpublished, and the result is not mentioned in [13]. Therefore, we shall include the proof of Theorem 1, using our methods which are quite different from those of Stallard, to illustrate the fact that even though at each step that we take, the "spread" that we shall discuss may be reduced to some extent, this does not matter as long as only finitely many steps (corresponding to the periodicity of a component) are taken. For a wandering domain, infinitely many steps are required, so that greater care needs to be taken, as we shall see in the proof of Theorem 2 .

Theorem 1 leaves open the possibility that a component $D$ of $N(f)$ is unbounded only if $D$ is a wandering domain in which the constant infinity is among the limit functions of convergent subsequences of iterates of $f$. When considering wandering domains, one can assume that they are simply connected, since by [4, Theorem 3.1, p. 565], if $f$ is any transcendental entire function such that $N(f)$ has a multiply connected component, then each component of $N(f)$ is bounded. Concerning this case Baker [3, Theorem 2, p. 484] proved that $D$ will still have to be bounded if

$$
\log M(r, f)=O\left((\log r)^{p}\right)
$$


as $r \rightarrow \infty$ where $1<p<3$. Here and later, we use the standard notations for the maximum modulus $M(r, f)$ and minimum modulus $m(r, f)$ of $f$, namely,

$$
\begin{gathered}
M(r, f)=\max \{|f(z)|:|z|=r\}, \\
m(r, f)=\min \{|f(z)|:|z|=r\} .
\end{gathered}
$$

Stallard [13, Theorem B, p. 43] improved the sufficient condition (1.1) to

$$
\log \log M(r, f)=O\left(\frac{(\log r)^{1 / 2}}{(\log \log r)^{\varepsilon}}\right)
$$

for some $\varepsilon>0$. Stallard [13, Theorem C, p. 44] proved furthermore that every component of $N(f)$ is bounded provided that $f$ is of order less than $1 / 2$ and

$$
\frac{\log M(2 r, f)}{\log M(r, f)} \rightarrow c \quad \text { as } r \rightarrow \infty
$$

where $c \geq 1$ is a finite constant that depends on $f$. It remains an open question what can happen to wandering domains, and what can happen to Baker domains of period greater than 1 when $f$ is exactly of order $1 / 2$, minimal type.

Our method of proof, based on the notion of "self-sustaining spread", yields also some results in the case of wandering domains $D$. Unfortunately we require a regularity condition on the growth of $f$. We believe this condition to be unnecessary, but our method of proof does not permit us to omit it.

We set $\varphi(x)=\log M\left(e^{x}, f\right)$ so that, by the Hadamard three-circles theorem, $\varphi(x)$ is an increasing convex function of $x$. The function $\varphi^{\prime}(x)$ may fail to exist at a countable set of points. At such points $\varphi^{\prime}(x)$ is understood to be the right-hand derivative.

Theorem 2. Let $f$ be a transcendental entire function of order $\rho<1 / 2$ such that for some positive constant $c$

$$
\frac{\varphi^{\prime}(x)}{\varphi(x)} \geq \frac{1+c}{x}
$$

for all sufficiently large $x$, where $\varphi(x)=\log M\left(e^{x}, f\right)$. Then every component of the set of normality $N(f)$ of $f$ is bounded.

The one-sided condition (1.4) should be compared to the condition

$$
\varphi(x+1) \sim c \varphi(x) \quad \text { as } x \rightarrow \infty
$$

which is Stallard's condition (1.3).

In conclusion we remark that (1.4) is a condition of growth as well as regularity. It is equivalent to the condition

$$
\liminf _{r \rightarrow \infty} \frac{d \log \log M(r, f)}{d(\log \log r)}>1
$$

which implies, in particular, that

$$
\log M(r, f)>(\log r)^{1+\delta}
$$

for some $\delta>0$ and all $r>r_{0}(\delta)$. The difficulty, therefore, in proving Theorem 2 in full generality by a method like ours, arises from the possible presence of large annuli which are almost zero free, where the growth of the function $f$ becomes very much like that of a polynomial. In such annuli a much stronger version of 
the $\cos \pi \rho$-theorem holds, and it seems reasonable to expect that the spread will sustain itself also in such regions.

We thank Professor I. N. Baker for his valuable comments.

\section{Auxiliary Results}

If $E \subset[1, \infty)$, the lower logarithmic density of the set $E$ is defined by

$$
\underline{\log \operatorname{dens}} E=\liminf _{R \rightarrow \infty} \frac{1}{\log R} \int_{E \cap(1, R)} \frac{d t}{t} .
$$

Of fundamental importance to our proofs is the $\cos \pi \rho$-theorem. The form of this most suitable for us is the following, which can be found in [5, p. 294].

Theorem B. Let $f$ be a transcendental entire function of order $\rho<1 / 2$, and suppose that $\rho<\alpha<1 / 2$. Then if

$$
E=\{r \geq 1: \log m(r, f)>(\cos \pi \alpha) \log M(r, f)\}
$$

we have

$$
\underline{\log \operatorname{dens}} E \geq 1-\frac{\rho}{\alpha}
$$

In particular, $\liminf \operatorname{in}_{r \rightarrow \infty} m(r, f)=\infty$.

If $f$ is of order $1 / 2$, minimal type, we may conclude only that $m(r, f)$ is unbounded. When $\rho<1 / 2$, we take $\alpha=(1+2 \rho) / 4$ so that if

$$
\beta=\cos \left(\pi \frac{1+2 \rho}{4}\right), \quad \sigma=\frac{2}{1-2 \rho}>\frac{1+2 \rho}{1-2 \rho},
$$

then, given any sufficiently large $R$, say $R \geq R_{0}$, we have

$$
\log m(r, f)>\beta \log M(r, f)
$$

for some $r$ in the range $R \leq r \leq R^{\sigma}$.

\section{Proof of Theorem 1}

Let $f$ be a transcendental entire function of order $\rho<1 / 2$, and suppose that $\Omega_{0}$ is a preperiodic or periodic component of $N(f)$. Thus there are minimal positive integers $k, p$ such that $f^{k}\left(\Omega_{0}\right) \subset \Omega_{1}$ and $f^{p}\left(\Omega_{1}\right) \subset \Omega_{1}$, where $\Omega_{1}$ is a component of $N(f)$. If $\Omega_{0}$ is unbounded, then so is $\Omega_{1}$, by Theorem B. Hence it suffices to prove that each periodic component of $N(f)$ is bounded.

Let $D$ be a periodic component of $N(f)$, and let $D=D_{0}, D_{1}, \ldots, D_{p-1}$ be the distinct components of $N(f)$ such that, with $D_{p}=D_{0}$, we have $f\left(D_{j}\right) \subset D_{j+1}$ for $0 \leq j \leq p-1$. By [3, Lemma 7, p. 490], $D$ is bounded unless some subsequence of iterates of $f$ tends to the constant infinity locally uniformly in $D$. Now any invariant component under $f^{p}$ is attracting, superattracting, parabolic, a Siegel disk, or a Baker domain (see, e.g., [7]) since, by the maximum modulus principle, entire functions cannot have Herman rings. If $\lim _{k \rightarrow \infty} f^{n_{k}}(z)=\infty$ locally uniformly in $D$ for some subsequence $n_{k} \rightarrow \infty$, then $D$ is a Baker domain and $\lim _{n \rightarrow \infty} f^{n}(z)=\infty$ locally uniformly in $D$. To get a contradiction, we suppose from now on that $D$ (and each $D_{j}$ ) is an unbounded Baker domain for $f^{p}$. If $p=1$, that is, if $D$ is invariant, this is impossible by [3, Theorem 6 , p. 485], so we assume that $p \geq 2$.

By $[4$, Theorem 3.1, p. 565], any multiply connected component of $N(f)$ is a wandering domain. Here $D$, and each $D_{j}$, is simply connected. Following the 
arguments in [3, proof of Theorem 6, p. 488], we find points $z_{00}, z_{01}=f^{p}\left(z_{00}\right) \in$ $D=D_{0}$ and a path $\gamma_{00} \subset D_{0}$ joining $z_{00}$ to $z_{01}$ with the following properties. Write

$$
\gamma_{j}=\bigcup_{k=0}^{\infty} f^{j+k p}\left(\gamma_{00}\right) \subset D_{j}
$$

for $0 \leq j<p$. Then each $\gamma_{j}$ is a path going to infinity, and $f^{m}\left(\gamma_{j}\right) \subset \gamma_{l}$ where $0 \leq l<p$ and $l \equiv m+j(\bmod p)$. In particular, $f^{p}\left(\gamma_{j}\right) \subset \gamma_{j}$ for all $j$. We set $z_{j k}=f^{j+k p}\left(z_{00}\right)$ and note that $z_{j k} \in D_{j}$ for $0 \leq j<p$ and $k \geq 1$. By [7, (7), p. 166], we have

$$
1 / C_{0} \leq\left|f^{p}(z)\right| /|z| \leq C_{0}
$$

for all $z \in \bigcup_{j=0}^{p-1} \gamma_{j}$, for some suitable constant $C_{0}>1$. (The open set $G$ in the proof of $[7,(7)$, p. 166] should be connected, but the proof shows that the union of finitely many simply connected domains, such as $\bigcup_{j=0}^{p-1} D_{j}$, will also do.) In particular, since $z_{j, k+1}=f^{p}\left(z_{j k}\right)$,

$$
1 / C_{0} \leq\left|z_{j, k+1}\right| /\left|z_{j k}\right| \leq C_{0}
$$

for all $j, k$. Taking $C_{0}$ large enough, we may assume that

$$
1 / C_{0} \leq|z| /|w| \leq C_{0}
$$

for all $z, w \in \gamma_{j 0}$, for each $j$ with $0 \leq j<p$. Then (3.3) holds for all $z, w \in \gamma_{j k}$, for all $j, k$, provided that $C_{0}$ is replaced by a suitable large constant, obtained as in the proof of (4.1) below, and again denoted by $C_{0}$.

We may assume that $\left|f^{j}(z)\right| \geq 3$ for all $j \geq 0$ and all $z \in \bigcup_{k=0}^{p-1} \gamma_{k}$. Further, $z_{j k} \rightarrow \infty$ as $k \rightarrow \infty$, for each $j$. This, together with (3.2), implies that, setting

$$
R_{j k}=\log \left|z_{j k}\right|>1
$$

we have

$$
1 / C \leq R_{j, k+1} / R_{j k} \leq C
$$

for all $j, k$, for some suitable $C>1$ (in fact, any $C>1$ would do if we restrict $k$ to be large enough, depending on $C$, but we shall not need such precision).

When necessary, we write $z_{j k}=z_{j-p, k+1}$ and $R_{j k}=R_{j-p, k+1}$ if $j \geq p$. If, for some $j$, the ratio

$$
\frac{R_{j+1, k}}{R_{j k}}=\frac{\log \left|f\left(z_{j k}\right)\right|}{\log \left|z_{j k}\right|}
$$

is bounded as $k \rightarrow \infty$, then by (3.3), we see that $|f(z)|=O\left(|z|^{q}\right)$ for some $q>0$, as $z \rightarrow \infty$ along $\gamma_{j}$. By Theorem $\mathrm{B}$, this is a contradiction. Hence we may assume that

$$
\limsup _{k \rightarrow \infty} \frac{R_{j+1, k}}{R_{j k}}=\infty
$$

for each $j$. Also

$$
\prod_{j=l}^{p-1+l} \frac{R_{j+1, k}}{R_{j k}}=\frac{R_{l, k+1}}{R_{l, k}}=\frac{\log \left|f^{p}\left(z_{l k}\right)\right|}{\log \left|z_{l k}\right|} \in[1 / C, C]
$$


for all $k \geq 0$ and all $l$ with $0 \leq l<p$. Hence for any $\varepsilon \in\left(0, C^{-4}\right)$, there are integers $l, k$ such that

$$
\frac{R_{l+1, k}}{R_{l k}}>\frac{C}{\varepsilon^{p-1}}
$$

and consequently there is an integer $j$ such that (for the same $k$ )

$$
\frac{R_{j+1, k}}{R_{j k}}<\varepsilon
$$

Here we may take $k$, and, hence, $R_{j k}$, to be arbitrarily large.

Take $Q$ to be the minimal positive integer such that

$$
\frac{R_{j+1, k+Q}}{R_{j, k+Q}}>1
$$

Such a number $Q$ exists since $z_{j m} \rightarrow \infty$ as $m \rightarrow \infty$, for each $j$, and since $\log |f(z)| / \log |z|$ is unbounded on each $\gamma_{j}$. By (3.4),

$$
\frac{R_{j+1, k+q+1} / R_{j, k+q+1}}{R_{j+1, k+q} / R_{j, k+q}}=\frac{R_{j+1, k+q+1} / R_{j+1, k+q}}{R_{j, k+q+1} / R_{j, k+q}} \in\left[C^{-2}, C^{2}\right]
$$

so that $Q>2$ since $\varepsilon<C^{-4}$. Further, by (3.4) we have

$$
1<\frac{R_{j+1, k+Q}}{R_{j, k+Q}} \leq C^{2}
$$

Recall the definition (2.1) of $\sigma$ and $\beta$. Suppose firstly that

$$
R^{\prime \prime} / R^{\prime} \geq \sigma
$$

where $R^{\prime \prime}=\max \left\{R_{j, k+q}: 0 \leq q \leq Q\right\}$ and $R^{\prime}=\min \left\{R_{j, k+q}: 0 \leq q \leq Q\right\}$. Then by (3.3), we find $z \in \gamma_{j}$ (with $z \in \bigcup_{q=0}^{Q-1} \gamma_{j, k+q}$ ) such that

$$
\log m(|z|, f)>\beta \log M(|z|, f) .
$$

There is an integer $\mu$ with $0 \leq \mu<Q$ such that

$$
\frac{1}{C} \leq \frac{\log |z|}{\log \left|z_{j, k+\mu}\right|} \leq C \quad \text { and } \quad \frac{1}{C} \leq \frac{\log |f(z)|}{\log \left|z_{j+1, k+\mu}\right|} \leq C .
$$

Hence, with $r=|z|$,

which gives

$$
C^{2}>\frac{R_{j+1, k+\mu}}{R_{j, k+\mu}} \geq C^{-2} \frac{\log |f(z)|}{\log |z|} \geq C^{-2} \beta \frac{\log M(r, f)}{\log r}
$$

$$
\log M(r, f) \leq C^{4} \beta^{-1} \log r .
$$

Since $f$ is transcendental, this gives a contradiction when $r$ is large enough, that is, when $R_{j k}$ is large enough. Thus we may suppose secondly that $R^{\prime \prime} / R^{\prime}<\sigma$.

If $S^{\prime \prime}=\max \left\{R_{j+1, k+q}: 0 \leq q \leq Q\right\}$ and $S^{\prime}=\min \left\{R_{j+1, k+q}: 0 \leq q \leq Q\right\}$, then

$$
\frac{S^{\prime \prime}}{S^{\prime}} \geq \frac{R_{j+1, k+Q}}{R_{j+1, k}} \geq \frac{R_{j, k+Q}}{\varepsilon R_{j k}} \geq \frac{R^{\prime}}{\varepsilon R^{\prime \prime}}>\frac{1}{\varepsilon \sigma} .
$$

Hence

$$
\frac{\max \left\{\log |z|: z \in \bigcup_{\mu=0}^{Q-1} \gamma_{j+1, k+\mu}\right\}}{\min \left\{\log |z|: z \in \bigcup_{\mu=0}^{Q-1} \gamma_{j+1, k+\mu}\right\}}>\frac{1}{\varepsilon \sigma} .
$$


We claim that whenever $\Gamma$ is a path such that $\log \left|\zeta_{2}\right| / \log \left|\zeta_{1}\right|>K_{0}>\sigma^{2}$ for some $\zeta_{1}, \zeta_{2} \in \Gamma$ and $\left|\zeta_{1}\right|>R_{0}$, where $R_{0}$ arises from (2.2) and is chosen to satisfy $M\left(R_{0}, f\right)^{\beta}>R_{0}$ as well, we have

$$
\frac{\log \left|f\left(\zeta_{4}\right)\right|}{\log \left|f\left(\zeta_{3}\right)\right|} \geq \frac{\beta K_{0}}{2 \sigma^{2}} \equiv A K_{0}
$$

and $\left|f\left(\zeta_{3}\right)\right|>R_{0}$ for some $\zeta_{3}, \zeta_{4} \in \Gamma$. Suppose that this has been proved. Applying this successively to $\Gamma=\bigcup_{\mu=0}^{Q-1} \gamma_{j+\nu, k+\mu}$ for $1 \leq \nu<p$, with $K_{0}=A^{\nu-1} /(\varepsilon \sigma)$, we find that there are $\zeta^{\prime}, \zeta^{\prime \prime} \in \bigcup_{\mu=1}^{Q} \gamma_{j, k+\mu}$ such that

$$
\frac{\log \left|\zeta^{\prime \prime}\right|}{\log \left|\zeta^{\prime}\right|}>\frac{A^{p-1}}{\varepsilon \sigma} \text {. }
$$

But then

$$
\frac{A^{p-1}}{\varepsilon \sigma}<C^{2} \frac{R^{\prime \prime}}{R^{\prime}}<C^{2} \sigma,
$$

which is a contradiction if $\varepsilon$ is chosen to be small enough.

We proceed to prove (3.6). This is what we mean by the concept of self-sustaining spread, even if, here, the "spread" can decrease by the factor $A$ each time. Now $\varphi(x)=\log M\left(e^{x}, f\right)$, and so, as previously mentioned, $\varphi$ is a strictly increasing convex function of $x$, defined on the real axis. We therefore have for $x>0$,

$$
\varphi(x)-\varphi(0)=\int_{0}^{x} \varphi^{\prime}(t) d t \leq \int_{0}^{x} \varphi^{\prime}(x) d t=x \varphi^{\prime}(x) .
$$

Thus

$$
\frac{d}{d x} \frac{\varphi(x)}{x}=\frac{x \varphi^{\prime}(x)-\varphi(x)}{x^{2}} \geq \frac{-\varphi(0)}{x^{2}}
$$

and so, whenever $0<x_{1}<x_{2}$,

$$
\frac{\varphi\left(x_{2}\right)}{x_{2}}-\frac{\varphi\left(x_{1}\right)}{x_{1}}=\int_{x_{1}}^{x_{2}}\left(\frac{d}{d x} \frac{\varphi(x)}{x}\right) d x \geq-\varphi(0)\left(\frac{1}{x_{1}}-\frac{1}{x_{2}}\right) \geq \min \left\{0, \frac{-\varphi(0)}{x_{1}}\right\} .
$$

When $x_{1}$ is large enough, we thus have

$$
\frac{\varphi\left(x_{2}\right)}{x_{2}}-\frac{\varphi\left(x_{1}\right)}{x_{1}} \geq \frac{-\varphi\left(x_{1}\right)}{2 x_{1}}
$$

so that

$$
\frac{\varphi\left(x_{2}\right)}{x_{2}} \geq \frac{\varphi\left(x_{1}\right)}{2 x_{1}}
$$

We could, of course, replace $1 / 2$ by any constant less than 1 . Hence, setting $\log r_{j}=$ $x_{j}$ for $j=1,2$, we have

$$
\frac{\log M\left(r_{2}, f\right)}{\log M\left(r_{1}, f\right)} \geq \frac{1}{2} \frac{\log r_{2}}{\log r_{1}}
$$

Given $\zeta_{1}, \zeta_{2} \in \Gamma$ with $\left|\zeta_{1}\right|>R_{0}$, and assuming that $\log \left|\zeta_{2}\right| / \log \left|\zeta_{1}\right|>K_{0}>\sigma^{2}$, we can find, by Theorem B, $r_{j}$ with $\left|\zeta_{1}\right| \leq r_{1} \leq\left|\zeta_{1}\right|^{\sigma}$ and $\left|\zeta_{2}\right|^{1 / \sigma} \leq r_{2} \leq\left|\zeta_{2}\right|$ such that 
$\log m\left(r_{j}, f\right)>\beta \log M\left(r_{j}, f\right)$ for $j=1,2$. There are points $\zeta_{3}, \zeta_{4} \in \Gamma$ with $\left|\zeta_{3}\right|=r_{1}$ and $\left|\zeta_{4}\right|=r_{2}$. We obtain

$$
\begin{aligned}
\frac{\log \left|f\left(\zeta_{4}\right)\right|}{\log \left|f\left(\zeta_{3}\right)\right|} & \geq \frac{\log m\left(r_{2}, f\right)}{\log M\left(r_{1}, f\right)} \geq \beta \frac{\log M\left(r_{2}, f\right)}{\log M\left(r_{1}, f\right)} \\
& =\beta \frac{\varphi\left(x_{2}\right)}{\varphi\left(x_{1}\right)} \geq \beta \frac{x_{2}}{2 x_{1}}>\frac{\beta K_{0}}{2 \sigma^{2}}
\end{aligned}
$$

and

$$
\begin{aligned}
\log \left|f\left(\zeta_{3}\right)\right| & \geq \log m\left(r_{1}, f\right)>\beta \log M\left(r_{1}, f\right) \\
& \geq \beta \log M\left(\left|\zeta_{1}\right|, f\right) \geq \beta \log M\left(R_{0}, f\right)>\log R_{0},
\end{aligned}
$$

as required. Thus (3.6) is proved, and the proof of Theorem 1 is complete.

\section{Proof of Theorem 2}

Let $f$ be as in Theorem 2, and let $D$ be an unbounded component of $N(f)$. In view of Theorem 1, $D$ must be a wandering domain. By [4, Theorem 3.1, p. 565], every component of $N(f)$ is simply connected. Let $D_{j}$ be the component of $N(f)$ containing $f^{j}(D)$, so that each $D_{j}$ is unbounded by Theorem B, and $D_{0}=D$.

If $K$ is a compact subset of $D$, then a modification of the proof of [7, (7), p. 166] shows that

$$
1 / C_{0} \leq\left|f^{j}(z)\right| /\left|f^{j}(w)\right| \leq C_{0}
$$

for all $z, w \in K$ and for all $j \geq 0$, for some suitable constant $C_{0}>1$. More precisely, we may conjugate $f$ by $a z+b$ for suitable complex constants $a, b$ with $a \neq 0$ so as to have

$$
\{z:|z| \leq 3\} \cap\left(\bigcup_{j=0}^{\infty} D_{j}\right)=\varnothing .
$$

Then the origin has a neighborhood that does not contain any limit function of any subsequence $f^{n_{k}}$ that converges locally uniformly in $D$ (as is well known, each such limit function is constant, possibly infinity). Let $L>1$ be a large constant, to be determined soon. Pick $j \geq 0$ and $z, w \in K$. Suppose that $\left|f^{j}(z)\right| /\left|f^{j}(w)\right|>L$. Let $\zeta \in \partial D_{j}$ have the smallest possible modulus, so that in particular, $|\zeta|<\left|f^{j}(w)\right|$. Let $h_{\Omega}\left(z_{1}, z_{2}\right)$ denote the hyperbolic distance between the points $z_{1}, z_{2}$ of the domain $\Omega$, and let $\lambda_{\Omega}(z)$ denote the density of the hyperbolic metric of $\Omega$ at $z \in \Omega$. Thus

$$
h_{D_{j}}\left(f^{j}(z), f^{j}(w)\right) \leq h_{D}(z, w) \leq L_{0} \equiv \max \left\{h_{D}\left(z_{1}, z_{2}\right): z_{1}, z_{2} \in K\right\} .
$$

Since $D_{j}$ is simply connected, we have, by Koebe's one-quarter theorem,

$$
\lambda_{D_{j}}(z) \geq \frac{1}{4 \operatorname{dist}\left(z, \partial D_{j}\right)} \geq \frac{1}{4|z-\zeta|} \geq \frac{1}{4(|z|+|\zeta|)}
$$


for all $z \in D_{j}$, where $\operatorname{dist}\left(z, \partial D_{j}\right)$ denotes the Euclidean distance of $z$ from $\partial D_{j}$. Hence

$$
\begin{aligned}
L_{0} & \geq h_{D_{j}}\left(f^{j}(z), f^{j}(w)\right) \geq \int_{\left|f^{j}(w)\right|}^{\left|f^{j}(z)\right|} \frac{d r}{4(r+|\zeta|)}=\frac{1}{4} \log \frac{\left|f^{j}(z)\right|+|\zeta|}{\left|f^{j}(w)\right|+|\zeta|} \\
& \geq \frac{1}{4} \log \frac{\left|f^{j}(z)\right|+\left|f^{j}(w)\right|}{2\left|f^{j}(w)\right|}=\frac{1}{4}\left(\log \left(1+\frac{\left|f^{j}(z)\right|}{\left|f^{j}(w)\right|}\right)-\log 2\right) \\
& \geq \frac{1}{4}(\log (1+L)-\log 2),
\end{aligned}
$$

which gives a contradiction if $L$ is sufficiently large if compared to $L_{0}$. This proves (4.1).

Suppose that $C$ is a preassigned constant subject only to $C>1$. Next we show that by (4.1) and (4.2), we have

$$
1 / C \leq \frac{\log \left|f^{j}(z)\right|}{\log \left|f^{j}(w)\right|} \leq C
$$

for all $z, w \in K$ and for all sufficiently large $j>0$. This replaces condition (3.4) of Theorem 1. For if (4.3) does not hold, then there are sequences $z_{j}, w_{j} \in K$ and integers $n_{j} \rightarrow \infty$ such that $\log \left|f^{n_{j}}\left(z_{j}\right)\right| / \log \left|f^{n_{j}}\left(w_{j}\right)\right|>C$, that is,

$$
\left|f^{n_{j}}\left(z_{j}\right)\right|>\left|f^{n_{j}}\left(w_{j}\right)\right|^{C} .
$$

By passing to a subsequence, we may assume that $\left|f^{n_{j}}\left(z_{j}\right)\right| \rightarrow R_{2}$ and $\left|f^{n_{j}}\left(w_{j}\right)\right| \rightarrow$ $R_{1}$, say, where $3 \leq R_{1}<R_{2}<\infty$ or $R_{1}=R_{2}=\infty$. In the former case, we do not have $f^{n_{j}} \rightarrow \infty$ locally uniformly in $D$, so that by passing to a further subsequence, we may assume that $f^{n_{j}} \rightarrow \omega$ locally uniformly in $D$, where $\omega$ is a complex number with $|\omega| \geq 3$ (by (4.2)). Hence $f^{n_{j}}\left(z_{j}\right) \rightarrow \omega$ and $f^{n_{j}}\left(w_{j}\right) \rightarrow \omega$ as $j \rightarrow \infty$, which contradicts (4.4). Thus $R_{1}=R_{2}=\infty$. But now, by (4.1), $\left|f^{n_{j}}\left(z_{j}\right)\right| \leq C_{0}\left|f^{n_{j}}\left(w_{j}\right)\right| \leq\left|f^{n_{j}}\left(w_{j}\right)\right|^{C}$ when $\left|f^{n_{j}}\left(w_{j}\right)\right|$ is large enough, which is a contradiction. This completes the proof of (4.3).

We now choose a real number $C$ with $1<C<4$. We may assume that (4.3) holds for $j \geq j_{0}$, say, for all $z, w \in K$. We have $\beta \log M(r, f)>\log r$ for all $r \geq R_{0}$, say. Suppose that (1.4) holds for all $x \geq x_{0}>1$. Then, if $x_{0}<x_{1}<x_{2}$, we have

$$
\log \frac{\varphi\left(x_{2}\right)}{\varphi\left(x_{1}\right)}=\int_{x_{1}}^{x_{2}} \frac{\varphi^{\prime}(x)}{\varphi(x)} d x \geq \int_{x_{1}}^{x_{2}} \frac{1+c}{x} d x=(1+c) \log \frac{x_{2}}{x_{1}}
$$

and so

$$
\frac{\varphi\left(x_{2}\right)}{\varphi\left(x_{1}\right)} \geq\left(\frac{x_{2}}{x_{1}}\right)^{1+c}
$$

Recall the definition (2.1) of $\sigma$ and $\beta$. Let $L>\sigma^{2}>4$ be a large constant satisfying also $\beta L^{c}>\sigma^{2(1+c)}$. Suppose that the compact connected subset $K$ of $D$ is chosen so that $\log |\zeta|>\max \left\{x_{0}, \log R_{0}\right\}$ for all $\zeta \in K$ and so that there are $z, w \in K$ with $\log |w|>L \log |z|$. This is possible since $D$ is unbounded. Write $K_{j}=f^{j}(K)$ so that $K_{j}$ is a compact connected subset of $D_{j}$.

It follows from Theorem B that there are $r_{1}, r_{2}$ with $|z| \leq r_{1} \leq|z|^{\sigma}$ and $|w|^{1 / \sigma} \leq$ $r_{2} \leq|w|$ such that $\log m\left(r_{j}, f\right)>\beta \log M\left(r_{j}, f\right)$ for $j=1,2$. Since $K$ is connected, there are points $\zeta_{1}, \zeta_{2} \in K$ with $\left|\zeta_{j}\right|=r_{j}$ for $j=1,2$. Write $x_{j}=\log r_{j}$ for $j=1,2$, 
so that $\log |z| \leq x_{1} \leq \sigma \log |z|$ and $\sigma^{-1} \log |w| \leq x_{2} \leq \log |w|$. We obtain

$$
\begin{aligned}
\frac{\log \left|f\left(\zeta_{2}\right)\right|}{\log \left|f\left(\zeta_{1}\right)\right|} & \geq \frac{\log m\left(r_{2}, f\right)}{\log M\left(r_{1}, f\right)} \geq \beta \frac{\log M\left(r_{2}, f\right)}{\log M\left(r_{1}, f\right)} \\
& =\beta \frac{\varphi\left(x_{2}\right)}{\varphi\left(x_{1}\right)} \geq \beta\left(\frac{x_{2}}{x_{1}}\right)^{1+c} \geq \beta\left(\frac{\sigma^{-1} \log |w|}{\sigma \log |z|}\right)^{1+c} \\
& >\beta\left(\frac{L}{\sigma^{2}}\right)^{1+c}>L .
\end{aligned}
$$

Also for $j=1,2$, we have

$\log \left|f\left(\zeta_{j}\right)\right| \geq \log m\left(r_{j}, f\right)>\beta \log M\left(r_{j}, f\right)>\log r_{j}=\log \left|\zeta_{j}\right|>\max \left\{x_{0}, \log R_{0}\right\}$.

Note that $\log r_{1}=x_{1} \geq \log |z|>\max \left\{x_{0}, \log R_{0}\right\}$ while, since $L>\sigma^{2}$, we have $\log r_{2}=x_{2} \geq \sigma^{-1} \log |w|>L \sigma^{-1} \log |z|>\log |z|>\max \left\{x_{0}, \log R_{0}\right\}$. We find that there are points $\zeta_{3}=f\left(\zeta_{1}\right)$ and $\zeta_{4}=f\left(\zeta_{2}\right)$ in $f(K)$ with $\log \left|\zeta_{j}\right|>\max \left\{x_{0}, \log R_{0}\right\}$ for $j=3,4$ and with

$$
\frac{\log \left|\zeta_{4}\right|}{\log \left|\zeta_{3}\right|}>L
$$

Now we may repeat the above argument and deduce that for all $j \geq 1$, there are $z, w \in f^{j}(K)$ with $\log |w|>L \log |z|$. Since $C<4<L$, we get a contradiction with (4.3) with $j \geq j_{0}$. This completes the proof of Theorem 2.

\section{REFERENCES}

1. I. N. Baker, Zusammensetzungen ganzer Funktionen, Math. Z. 69 (1958), 121-163. MR 20:4000

2. __ Fixpoints and iterates of entire functions, Math. Z. 71 (1959), 146-153. MR 21:5743

3. Soc. (Series A) 30 (1981), 483-495. MR 82g:30043

4. W Wandering domains in the iteration of entire functions, Proc. London Math. Soc. (Series 3) 49 (1984), 563-576. MR 86d:58066

5. P. D. Barry, On a theorem of Besicovitch, Quart. J. Math. Oxford (Series 2) 14 (1963), 292-302. MR 28:234

6. A. F. Beardon, Iteration of rational functions, Springer, New York, 1991. MR 92j:30026

7. W. Bergweiler, The iteration of meromorphic functions, Bull. Amer. Math. Soc. 29 (1993), 151-188.

8. P. Fatou, Sur les équations fonctionnelles, Bull. Soc. Math. France 47 (1919), 161-271.

9. Sur les équations fonctionnelles, Bull. Soc. Math. France 48 (1920), 33-94, 208-314.

10. - Sur l'itération des fonctions transcendantes entières, Acta Math. 47 (1926), 337-370.

11. G. Julia, Mémoire sur l'itération des fonctions rationnelles, J. Math. Pures Appl. 8 (1918), $47-245$.

12. G. Stallard, Some problems in the iteration of meromorphic functions, Ph.D. thesis, Imperial College, London, 1991.

13. - The iteration of entire functions of small growth, Math. Proc. Camb. Phil. Soc. 114 (1993), 43-55. MR 95a:30023

Department of Mathematics, University College, London WC1E 6BT, United KingDOM

Department of Mathematics, University of Illinois at Urbana-Champaign, Urbana, ILLINOIS 61801 